\title{
The efficacy and limits of magnetic resonance-guided focused ultrasound pallidotomy for Parkinson's disease: a Phase I clinical trial
}

\author{
Na Young Jung, MD, ${ }^{1}$ Chang Kyu Park, MD, ${ }^{3}$ Minsoo Kim, MD, ${ }^{1}$ Phil Hyu Lee, MD, PhD, ${ }^{2}$ \\ Young Ho Sohn, MD, PhD, ${ }^{2}$ and Jin Woo Chang, MD, PhD ${ }^{1}$
}

Departments of ${ }^{1}$ Neurosurgery and ${ }^{2}$ Neurology, Brain Research Institute, Yonsei University College of Medicine, Seoul; and ${ }^{3}$ Department of Neurosurgery, Kyung Hee University College of Medicine, Seoul, Republic of Korea

\begin{abstract}
OBJECTIVE Recently, MR-guided focused ultrasound (MRgFUS) has emerged as an innovative treatment for numerous neurological disorders, including essential tremor, Parkinson's disease (PD), and some psychiatric disorders. Thus, clinical applications with this modality have been tried using various targets. The purpose of this study was to determine the feasibility, initial effectiveness, and potential side effects of unilateral MRgFUS pallidotomy for the treatment of parkinsonian dyskinesia.
\end{abstract}

METHODS A prospective, nonrandomized, single-arm clinical trial was conducted between December 2013 and May 2016 at a single tertiary medical center. Ten patients with medication-refractory, dyskinesia-dominant PD were enrolled. Participants underwent unilateral MRgFUS pallidotomy using the Exablate 4000 device (InSightec) after providing written informed consent. Patients were serially evaluated for motor improvement, neuropsychological effects, and adverse events according to the 1-year follow-up protocol. Primary measures included the changes in the Unified Parkinson's Disease Rating Scale (UPDRS) and Unified Dyskinesia Rating Scale (UDysRS) scores from baseline to 1 week, 1 month, 3 months, 6 months, and 1 year. Secondary measures consisted of neuropsychological batteries and quality of life questionnaire (SF-36). Technical failure and safety issues were also carefully assessed by monitoring all events during the study period.

RESULTS Unilateral MRgFUS pallidotomy was successfully performed in 8 of 10 patients (80\%), and patients were followed up for more than 6 months. Clinical outcomes showed significant improvements of $32.2 \%$ in the "medicationoff" UPDRS part III score $(p=0.018)$ and $52.7 \%$ in UDysRS $(p=0.017)$ at the 6 -month follow-up, as well as $39.1 \%(p=$ $0.046)$ and $42.7 \%(p=0.046)$ at the 1 -year follow-up, respectively. These results were accompanied by improvement in quality of life. Among 8 cases, 1 patient suffered an unusual side effect of sonication; however, no patient experienced persistent aftereffects.

CONCLUSIONS In the present study, which marks the first Phase I pilot study of unilateral MRgFUS pallidotomy for parkinsonian dyskinesia, the authors demonstrated the efficacy of pallidal lesioning using MRgFUS and certain limitations that are unavoidably associated with incomplete thermal lesioning due to technical issues. Further investigation and long-term follow-up are necessary to validate the use of MRgFUS in clinical practice.

Clinical trial registration no.: NCT02003248 (clinicaltrials.gov)

https://thejns.org/doi/abs/10.3171/2018.2.JNS172514

KEYWORDS dyskinesia; magnetic resonance imaging; pallidotomy; Parkinson disease; focused ultrasound; functional neurosurgery

$\mathrm{S}$ INCE Laitinen and colleagues reestablished the beneficial effect of lesioning the posteroventral portion of the globus pallidus (pallidotomy), pallidotomy has been highlighted as an effective treatment for the cardinal motor symptoms of Parkinson's disease (PD) and levodopa-induced dyskinesia, especially when compared with other available medical therapies..$^{15,21,35}$ Recently, deep brain stimulation (DBS) of the subthalamic nucleus has become a mainstream neurosurgical technique for managing PD, with proven superiority for treating the dopaminer-

ABBREVIATIONS DBS = deep brain stimulation; ET = essential tremor; GPi = globus pallidus interna; MRgFUS = MR-guided focused ultrasound; PD = Parkinson's disease; RF = radiofrequency; UDysRS = Unified Dyskinesia Rating Scale; UPDRS = Unified Parkinson's Disease Rating Scale.

SUBMITTED October 5, 2017. ACCEPTED February 23, 2018.

INCLUDE WHEN CITING Published online August 10, 2018; DOI: 10.3171/2018.2.JNS172514. 
gic motor features of PD-like fluctuations or dyskinesia, and reducing the levodopa-equivalent dose. ${ }^{15,20}$ Despite these successful outcomes, DBS has limitations, including complications related to device implantation or electrical stimulation and relatively higher maintenance costs. Additionally, DBS requires continuous follow-up to check the device or to modulate stimulation parameters. These limitations make it unsuitable to apply DBS in patients who are immunocompromised or are in situations that make follow-up management difficult. ${ }^{34}$ Therefore, lesioning procedures may gain clinical importance as a substitute for or complement to DBS in some cases, or they can be a better option in individualized situations with clinical, social, and financial benefits. ${ }^{6}$ Despite its cost-effectiveness, pallidal lesioning by conventional radiofrequency (RF) techniques is less preferred, because of the risks of uncontrollable lesion size and consequent irreversible side effects, including hemorrhage, visual field deficits, and motor weakness. ${ }^{3,4,9,16,22}$ If these issues are addressed, lesioning procedures could play a more practical role in functional neurosurgery.

Recently, MR-guided focused ultrasound (MRgFUS) has come to be used as a minimally invasive surgical technique or nonincisional surgery. This technique can create more precise thermal lesions in deep brain tissue without requiring opening of the cranium. Precise location and volume of the lesion with MRgFUS can be monitored and controlled by real-time thermometry. ${ }^{18,25,26,28}$ Favorable clinical outcomes of MRgFUS ablation have been reported in several clinical trials involving patients with essential tremor (ET), obsessive-compulsive disorder, and intractable pain., ${ }^{7,12,13,17,19,23}$ In particular, MRgFUS thalamotomy for ET has recently been approved by the United States Food and Drug Administration because of its efficacy and safety. ${ }^{13}$ For PD, unilateral pallidothalamic tractotomy using MRgFUS was first utilized for the alleviation of tremor-dominant motor symptoms, resulting in a mean reduction of $60.9 \%$ in Unified Parkinson's Disease Rating Scale (UPDRS) scores, after repeated peak energy application to the target area. ${ }^{25}$ Previously, we reported the successful use of unilateral MRgFUS pallidotomy in a patient with idiopathic PD. ${ }^{30}$ In the present study, we evaluated the 1-year follow-up outcomes of unilateral MRgFUS pallidotomy in 10 patients to determine whether it is feasible to make solid and effective lesions in the globus pallidus interna (GPi). Additionally, its clinical effectiveness and side effects were also assessed in patients with PD, particularly with a focus on improving dyskinesia.

\section{Methods}

\section{Patient Selection and Study Design}

Between December 2013 and May 2016, 10 patients with idiopathic PD underwent unilateral MRgFUS pallidotomy at our institution. Patients between 20 and 80 years of age who had been diagnosed with PD more than 5 years previously by a neurologist specializing in movement disorders were included. The main enrollment criterion was medically refractory PD with uncontrolled motor fluctuation or levodopa-induced dyskinesia. All included patients exhibited an unequivocal response to dopaminer-
TABLE 1. Patient inclusion and exclusion criteria

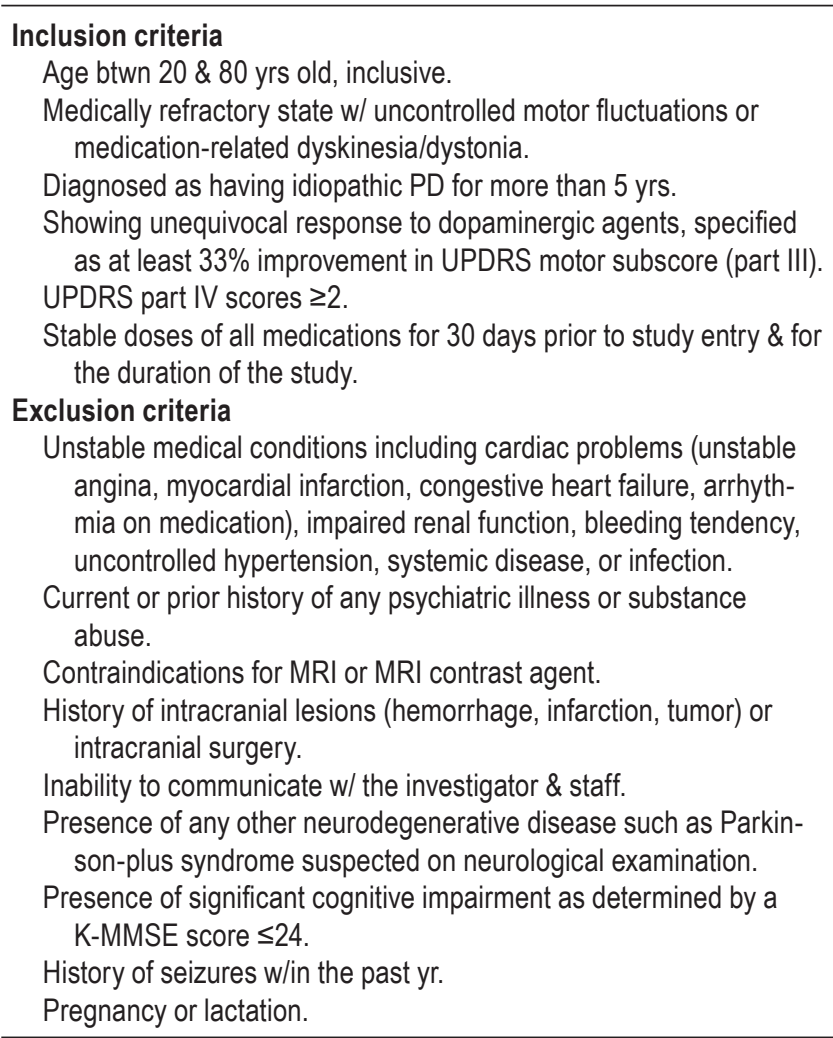

K-MMSE = Korean version of the Mini-Mental State Examination. Inclusion and exclusion criteria were agreed on by 2 members of the medical team.

gic agents, with at least $33 \%$ improvement in UPDRS motor subscores (part III) relative to the medication-off state. Patients were not eligible if they had been diagnosed with any psychiatric illness and/or other neurodegenerative diseases, such as Parkinson-plus syndromes. Those with unstable medical conditions, such as cardiac abnormalities, coagulopathy, renal disease, or substance abuse, were also excluded. In addition, contraindications for MRI or MRI contrast, as well as patients with intracranial lesions, were omitted from this study. The inclusion and exclusion criteria are listed in Table 1.

Clinical outcomes were evaluated by the same examiner using the UPDRS and Unified Dyskinesia Rating Scale (UDysRS) at baseline and 1 week, 1 month, 3 months, 6 months, and 12 months after treatment. A full battery of neuropsychological tests was used to assess perioperative alterations in multiple cognitive functions. Quality of life was evaluated using the SF-36 Physical Component Summary and Mental Component Summary scores. Imaging changes were assessed using various MRI sequences, including T1-weighted imaging (with and without contrast enhancement), T2-weighted imaging, diffusion-weighted imaging, and FLAIR sequences. Such standard clinical assessments and serial imaging workup were performed in accordance with the examination schedule presented in Table 2. During the follow-up period, neurological examinations were conducted to monitor for any adverse effects. 
TABLE 2. Study visit schedule and procedures

\begin{tabular}{|c|c|c|c|c|c|c|c|c|}
\hline \multirow[b]{2}{*}{ Parameter } & \multicolumn{8}{|c|}{ Visit } \\
\hline & $\begin{array}{l}\text { Screening } \\
\text { (-30 days) }\end{array}$ & $\begin{array}{c}\text { Pallidotomy } \\
\text { (0 day) }\end{array}$ & $\begin{array}{c}1 \text { Day } \\
( \pm 3 \text { days })\end{array}$ & $\begin{array}{c}1 \text { Wk } \\
( \pm 5 \text { days })\end{array}$ & $\begin{array}{c}1 \text { Mo } \\
( \pm 5 \text { days })\end{array}$ & $\begin{array}{c}3 \text { Mos } \\
( \pm 5 \text { days })\end{array}$ & $\begin{array}{c}6 \text { Mos } \\
( \pm 5 \text { days })\end{array}$ & $\begin{array}{c}12 \text { Mos } \\
( \pm 5 \text { days })\end{array}$ \\
\hline Written consent & 0 & & & & & & & \\
\hline History taking & 0 & & & & & & & \\
\hline CT scan & 0 & & & & & & & \\
\hline MRI & 0 & 0 & 0 & 0 & 0 & 0 & 0 & 0 \\
\hline Physical exam & 0 & & 0 & & & & & \\
\hline Neurological exam & 0 & 0 & 0 & 0 & 0 & 0 & 0 & 0 \\
\hline Visual field exam & 0 & & 0 & 0 & 0 & 0 & 0 & 0 \\
\hline UPDRS & 0 & & & 0 & 0 & 0 & 0 & 0 \\
\hline UDysRS & 0 & & & 0 & 0 & 0 & 0 & 0 \\
\hline SF-36 & 0 & & & & & 0 & 0 & 0 \\
\hline Neuropsychological test & 0 & & & & & & 0 & 0 \\
\hline Adverse events & & 0 & 0 & 0 & 0 & 0 & 0 & 0 \\
\hline
\end{tabular}

Written informed consent was obtained from the included patients prior to all procedures. This study received full approval from the ethical committee of the Korean Food and Drug Administration and our institutional review board. Details regarding this clinical trial are registered on clinicaltrials.gov (registration no. NCT02003248).

\section{Surgical Procedure for MRgFUS}

Conventional MRgFUS was performed at the left posteroventral GPi according to a previously described protocol. ${ }^{13,14}$ The entire procedure was performed using a 3-T MRI scanner (GE Medical Systems) using the Exablate 4000 device (InSightec). The entire scalp was completely shaved, and a scalp nerve block was performed to prevent the experience of pain during the cooling stage. ${ }^{20} \mathrm{~A}$ Cosman-Roberts-Wells stereotactic frame (Radionics) was then fixed on the patient's head under local anesthesia. A flexible silicone membrane with a central hole was applied to the patient's head to seal the space between the head and the transducer. This space contained cool, degassed water $\left(15^{\circ} \mathrm{C}-20^{\circ} \mathrm{C}\right)$ to reduce heating of the scalp. Prior to initiation of treatment, a series of standard diagnostic MRI scans were obtained to identify the location requiring treatment. The target area of the left GPi included the following coordinates: $20 \mathrm{~mm}$ lateral to the midline, $3-4 \mathrm{~mm}$ anterior to the midpoint between the anterior and posterior commissures, and 3-4 $\mathrm{mm}$ inferior to the intercommissural line.

Prior to producing permanent thermal lesions, subthreshold low-power sonication was applied several times for 10-20 seconds to reach a peak temperature between $40^{\circ} \mathrm{C}$ and $42^{\circ} \mathrm{C}$. This preliminary sonication allowed us to evaluate the exact location and size of the thermal spot and determine the appropriate sonication parameters. Highpower sonication was then delivered to the target area under the guidance of MRI and MR thermometry. Acoustic power and energy were steadily increased until target temperatures over $54^{\circ} \mathrm{C}$ had been achieved. We aimed to induce 2 or 3 separate lesions that were elliptical in shape.
During the entire series of sonication, the patient was examined repeatedly to assess neurological status. These assessments included monitoring for contralateral rigidity and bradykinesia, asking the patient to repeat several complex words in order to assess speech, and reporting of phosphenes and similar phenomena. When necessary, pain medication was provided to reduce back pain from lying in stationary position. All patients remained awake and responsive throughout the procedure, after which they were fully monitored for 1 day in the hospital.

\section{Statistical Analysis}

All statistical analyses were performed using IBM SPSS (version 23, IBM Corp.). Wilcoxon's signed-rank test was used for simple comparisons of the sizes of the matched preoperative and postoperative scores. Any differences in clinical characteristics and postoperative scoring between groups were analyzed by either t-tests or Fisher's exact tests as appropriate. The threshold for statistical significance was set at $p<0.05$. A repeated-measures ANOVA was also used to analyze the data for within-subject correlation. The statistical significance level in the posttest was corrected using Bonferroni's method.

\section{Results \\ Patient Characteristics and Clinical Outcomes}

The mean age of all participants was 59.8 years (range 52-73 years). There were 4 men and 6 women. The average duration of disease was 10.1 years (range 6-14 years). The preoperative levodopa equivalent dose was $1156 \mathrm{mg} /$ day (range 738-1750 mg/day). All participants underwent left pallidotomy with MRgFUS. The ultrasonic energy used for ablation ranged from $1500 \mathrm{~J}$ to $33,840 \mathrm{~J}$. The number of sonications for the entire lesioning procedure ranged from 10 to 27 , with durations of 10-31 seconds, and the overall procedure lasted 3-5 hours. The mean maximal temperature achieved in the MRgFUS treatments was $54.9^{\circ} \mathrm{C}$. Sonication-related data and the results of MRgFUS lesion- 
TABLE 3. Sonication-related information and technical results of MRgFUS

\begin{tabular}{rccccccc}
\hline $\begin{array}{c}\text { Case } \\
\text { No. }\end{array}$ & $\begin{array}{c}\text { Skull Density } \\
\text { Ratio }\end{array}$ & $\begin{array}{c}\text { Skull Vol } \\
\left(\mathrm{mm}^{3}\right)\end{array}$ & $\begin{array}{c}\text { No. of } \\
\text { Sonications }\end{array}$ & $\begin{array}{c}\text { Range of Energy } \\
\text { Delivered }(\mathrm{J})\end{array}$ & $\begin{array}{c}\text { Mean Peak } \\
\text { Focal Temp }\left({ }^{\circ} \mathrm{C}\right)\end{array}$ & $\begin{array}{c}\text { No. of Sonications } \\
>54^{\circ} \mathrm{C}\end{array}$ & \begin{tabular}{c} 
Results of Procedure \\
\hline 1
\end{tabular} \\
\hline 0.53 & 297 & 21 & $1500-16,500$ & 59 & 6 & Complete, visible lesion \\
\hline 2 & 0.47 & 394 & 16 & $1500-10,925$ & 44 & 5 & Incomplete, invisible lesion \\
\hline 3 & 0.51 & 289 & 27 & $2500-27,550$ & 56 & 3 & Complete, visible lesion \\
\hline 4 & 0.51 & 331 & 25 & $1500-33,840$ & 54 & 7 & Complete, visible lesion \\
\hline 5 & 0.62 & 333 & 22 & $1500-25,625$ & 58 & 3 & Complete, visible lesion \\
\hline 6 & 0.47 & 299 & 25 & $2010-32,000$ & 54 & 7 & Complete, visible lesion \\
\hline 7 & 0.54 & 273 & 24 & $2010-25,389$ & 57 & 2 & Complete, visible lesion \\
\hline 8 & 0.48 & 270 & 10 & $3000-18,700$ & 58 & 8 & Complete, visible lesion \\
\hline 9 & 0.63 & 292 & 24 & $1500-13,200$ & 61 & 48 & Complete, visible lesion \\
\hline 10 & 0.55 & 313 & 21 & $2500-25,536$ & 48 & Incomplete, invisible lesion \\
\hline
\end{tabular}

Temp $=$ temperature.

ing are shown in Table 3. Immediately after the procedure, 2 patients were excluded from the study as the following could not be attained: sufficient temperature rise and any visible lesion on the temperature map on MR thermometry despite repeated attempts or on MRI performed after the ablation. During the follow-up period, 2 additional patients did not finish the entire planned assessments after 6 months, as one patient did not cooperate with follow-up evaluation due to personal emotional issues, and the other was not properly evaluated due to severe back pain caused by lumbar fracture unrelated to MRgFUS pallidotomy. As a result, the clinical outcome of this trial was analyzed using data of 8 patients who finished the pallidotomy for 6-month follow-up, while data of 6 patients who reached the final follow-up were used to analyze results for 1-year follow-up. The mean maximal temperature of the included patients was $57.1^{\circ} \mathrm{C}$.

Prior to surgery, the average UPDRS scores were 1.9 for part I, 5.8 for part II, 30.4 for "medication-off" part III, 8.5 for "medication-on" part III, and 10.1 for part IV. Although varying results were observed during the follow-up period, improvements in all average scores were observed at 6 months after pallidotomy: $1.1(42.1 \%)$ for part I, $4.0(31.0 \%)$ for part II, 20.6 (32.2\%) for "medication-off" part III, 7.4 (12.9\%) for "medication-on" part III, and $4.3(57.4 \%)$ for part IV. UPDRS scores at the 1-year follow-up in 6 patients also improved: 1.5 (21.1\%) for part I, $3.6(39.7 \%)$ for part II, 18.5 (39.1\%) for "medication-off" part III, $5.8(31.8 \%)$ for "medication-on" part III, and 5.0 $(50.5 \%)$ for part IV. In particular, UPDRS "medicationoff" part III and part IV scores at each follow-up assessment showed significant improvement compared with baseline scores $(\mathrm{p}<0.05)$. The mean total UDysRS score also decreased by $52.7 \%$ at 6 months (from 41.9 to 19.8 , $\mathrm{p}=0.017$ ), and by $42.7 \%$ at the final follow-up of 1 year (from 41.9 to 24.0, $\mathrm{p}=0.046$ ) (Fig. 1).

The full battery of neuropsychological tests was conducted at baseline, and 6 and 12 months after pallidotomy (Table 4). When compared with baseline values, no significant changes were observed for most factors, with the exception of visual memory recognition and semantic generative naming. For quality of life, the average im- provement in the Physical Component Summary score between baseline and final follow-up was 6.22 points, while improvement in the Mental Component Summary score was 1.89 points.

\section{MRI Changes on Follow-Up Imaging}

Preoperative CT and MRI showed no abnormal findings. Follow-up MRI scans were obtained immediately after MRgFUS treatments and serially acquired at 1 day, 1 week, 1 month, 3 months, 6 months, and 12 months after MRgFUS pallidotomy. The morphological pattern of destructive lesions of the left GPi was similar in all patients. Signal changes on T2- and T1-weighted images were visible immediately after the procedure and remained prominent until 1 month after treatment, due to perilesional edema and blood-brain barrier disruption, which gradually disappeared beginning at the 3-month follow-up (Fig. 2). Volume changes in the lesions after MRgFUS pallidotomy demonstrated the peak volume enlargement at 1 week after treatment, then slowly decreased by 6 months (Fig. 3). A slightly increased volume shown on the 1-year follow-up MRI is presumed to be a scar change of the lesion site.

\section{Adverse Events}

All patients experienced mild headache while they received sonication in the MR machine and pin-site pain after frame removal, for which medication was not usually necessary to relieve pain. Four patients reported experiencing back pain arising from lying in a fixed position, although the pain subsided following administration of an analgesic. Neurological and psychological deficits during or after the MRgFUS procedure were absent in all but 1 patient, a 66-year-old man (case 8). When the 10th sonication was performed to deliver energy through the skull, the patient suddenly experienced dysarthria and grade III right motor hemiparesis. MRI revealed abnormally high signal intensity in the left anterior thalamus near the internal capsule, separate from the original target lesion, presumably due to heating injury (Fig. 4). Fortunately, he recovered completely within 2 days and experienced 

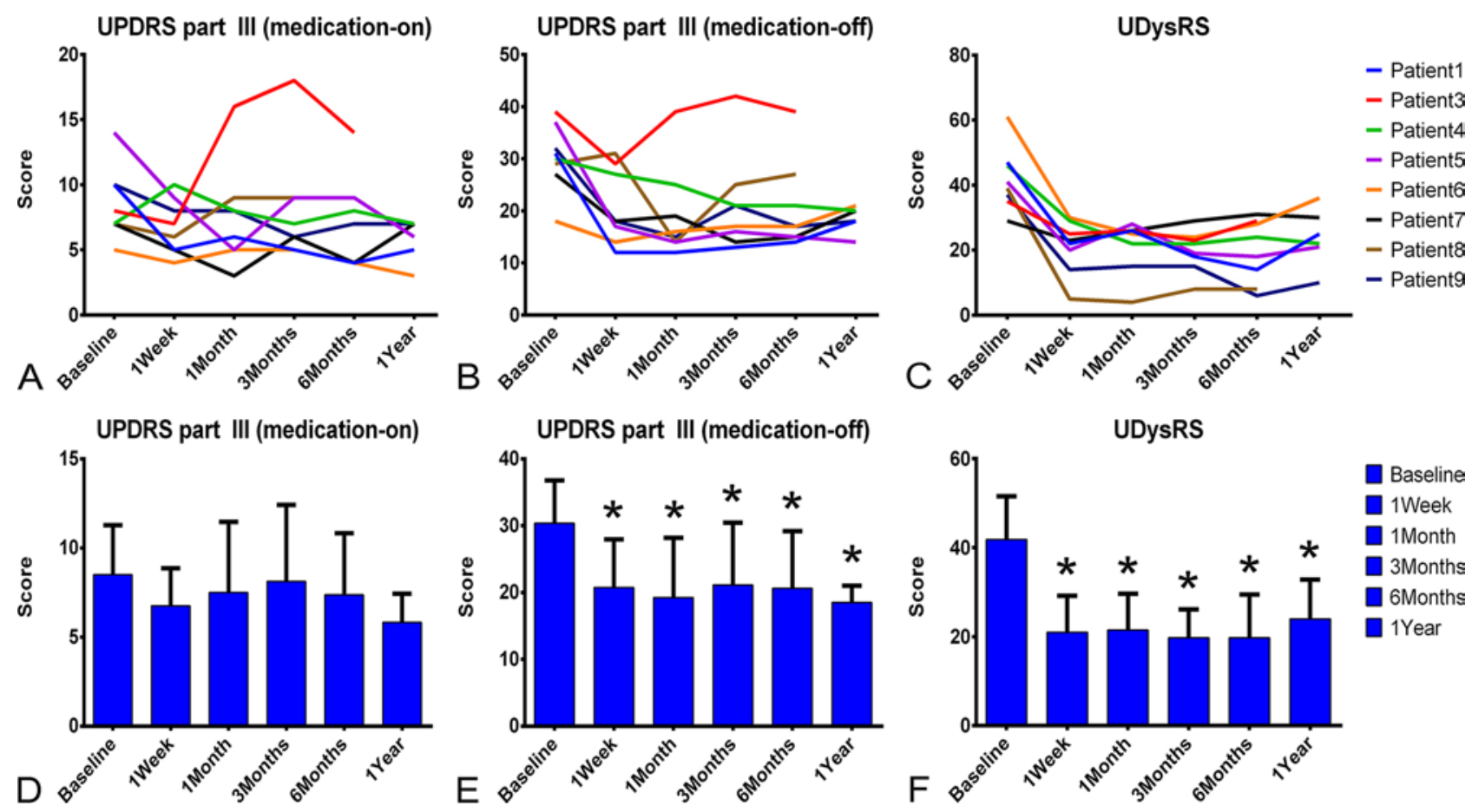

FIG. 1. Improvement in motor function following MRgFUS pallidotomy as assessed by clinical scales. UPDRS part III (medicationon and -off states) and UDysRS. UPDRS "medication-on" part III (A) and "medication-off" part III (B) scores for each patient showed varying results. UDysRS (C) had a general tendency of improvement after treatment. Unlike in "medication-on" UPDRS part III (D), the mean value of each motor score demonstrated significant improvement in "medication-off" part III (E) and UDysRS (F) at each point of follow-up evaluation. One-year follow-up outcome signified data from 8 patients, except 2 patients who dropped out during the middle of follow-up. * Statistically significant decrease in scores compared with baseline. The unusual results of the UPDRS part III score in case 3 may have resulted from a scoring problem due to patient's lack of cooperation. Figure is available in color online only.

improvements in PD-related symptoms, such as severe dyskinesia of the right arm. Afterward, he was followed up for safety, and no further deterioration was observed. His dyskinesia also improved, but other motor symptoms did not seem to improve, because of immobility, which was caused by worsened back pain from existing lumbar disease.

\section{Discussion}

Since high-intensity focused ultrasound was first combined with stereotactic neurosurgical procedures in 2009, MRgFUS has been widely used in patients with neurological conditions, such as ET, neuropathic pain, obsessivecompulsive disorder, and PD. 5, , 13,19,23,25,26 Compared with other surgical modalities, the most important advantages of MRgFUS pallidotomy are the noninvasiveness and accurate controlling of location and size of the target using real-time MRI and MR thermometry. ${ }^{18,25,26,28}$ Noninvasive procedures can reduce the risk of infection related to hardware implantation and shorten the hospital stay. In addition, MRgFUS does not require the use of anesthetic agents during sonication and can thus be safely performed in patients who have high surgical risks related to general anesthesia. To increase the applicability in clinical practice, we attempted to create lesions in the GPi using the same MRgFUS protocol adopted for patients with ET in our previous study. To our knowledge, this is the first clin- ical trial in which MRgFUS posteroventral pallidotomy for PD has been performed.

In comparison to RF ablation, MRgFUS usually generates a relatively low temperature increase in the target area. Although there is still no consensus to determine what temperature threshold is necessary to cause permanent tissue necrosis for the brain, previous clinical evidence has shown that a peak temperature of $54^{\circ} \mathrm{C}-60^{\circ} \mathrm{C}$ is sufficient to complete lesioning with less risk of bleeding. ${ }^{17,25,36} \mathrm{~A}$ recent study reported that an irreversible electrophysiological change in cardiac tissue could be induced when focal temperature increased above $52.3^{\circ} \mathrm{C} \pm 1.4^{\circ} \mathrm{C}$ using high-intensity focused ultrasound. ${ }^{37}$ Brain tissue is well known to be more thermally sensitive than other parts of the body, and its threshold for permanent lesioning can be low. ${ }^{11}$ In this study, a maximum temperature of less than $50^{\circ} \mathrm{C}$ was unable to form tissue damage. Subsequent analysis revealed that MRgFUS pallidotomy was associated with beneficial effects on motor improvement, which led to a reduction of $32.2 \%$ at 6 months and $39.1 \%$ at 1 year in the "medication-off" UPDRS part III score. This result is comparable to the existing success rate of $28.8 \%-37 \%$ reduction using conventional RF pallidotomy or unilateral GPi stimulation. . $^{10,27,31,33,35}$ In terms of levodopa-induced dyskinesia, score reduction in UPDRS part IV scores by $57.4 \%$ and UDysRS scores by $52.7 \%$ at 6 months established that MRgFUS pallidotomy could play a role in controlling dyskinesia compared with conventional RF palli- 
TABLE 4. Results of neuropsychological test following MRgFUS pallidotomy

\begin{tabular}{|c|c|c|c|c|}
\hline Factors & Baseline & 6-Mo FU & 12-Mo FU & $\mathrm{p}$ Value \\
\hline K-MMSE score & $27.83 \pm 0.75$ & $27.83 \pm 0.98$ & $26.83 \pm 1.60$ & 0.396 \\
\hline \multicolumn{5}{|l|}{ Attention } \\
\hline Digit span (forward) & $6.50 \pm 1.22$ & $6.00 \pm 1.55$ & $6.50 \pm 1.22$ & 0.305 \\
\hline Digit span (backward) & $4.17 \pm 0.75$ & $4.00 \pm 0.63$ & $3.67 \pm 1.03$ & 0.634 \\
\hline Digit span (forward-backward) & $2.33 \pm 1.03$ & $2.00 \pm 1.79$ & $2.83 \pm 0.98$ & 0.434 \\
\hline \multicolumn{5}{|l|}{ Language \& related function } \\
\hline K-BNT & $49.50 \pm 5.47$ & $52.17 \pm 4.17$ & $51.33 \pm 5.57$ & 0.273 \\
\hline Repetition & $14.67 \pm 0.82$ & $14.50 \pm 1.22$ & $14.83 \pm 0.41$ & 0.374 \\
\hline Calculation & $11.83 \pm 0.41$ & $11.50 \pm 0.84$ & $10.67 \pm 1.86$ & 0.100 \\
\hline \multicolumn{5}{|l|}{ Visuospatial function } \\
\hline CDT & $2.50 \pm 1.22$ & $3.00 \pm 0.00$ & $2.83 \pm 0.41$ & 0.525 \\
\hline RCFT & $32.00 \pm 5.22$ & $30.67 \pm 6.86$ & $28.33 \pm 8.24$ & 0.283 \\
\hline \multicolumn{5}{|l|}{ Verbal memory function (SVLT) } \\
\hline Immediate recall & $19.17 \pm 5.49$ & $20.17 \pm 5.42$ & $18.17 \pm 7.31$ & 0.437 \\
\hline Delayed recall & $6.00 \pm 2.83$ & $7.33 \pm 2.07$ & $7.00 \pm 3.16$ & 0.397 \\
\hline Recognition & $21.33 \pm 2.58$ & $20.17 \pm 1.94$ & $20.00 \pm 2.37$ & 0.188 \\
\hline \multicolumn{5}{|l|}{ Visual memory function (RCFT) } \\
\hline Immediate recall & $17.33 \pm 7.94$ & $19.33 \pm 8.54$ & $19.25 \pm 6.77$ & 0.557 \\
\hline Delayed recall & $16.75 \pm 7.45$ & $17.33 \pm 6.05$ & $18.67 \pm 7.08$ & 0.224 \\
\hline Recognition & $21.67 \pm 1.63$ & $19.33 \pm 1.21$ & $19.67 \pm 1.50$ & 0.006 \\
\hline \multicolumn{5}{|l|}{ Frontal executive function } \\
\hline Contrasting program & $18.33 \pm 4.08$ & $20.00 \pm 0.00$ & $19.50 \pm 0.84$ & 0.459 \\
\hline Go-no-go test & $16.67 \pm 8.16$ & $18.17 \pm 4.02$ & $14.00 \pm 8.51$ & 0.624 \\
\hline Semantic generative naming & $34.67 \pm 5.65$ & $25.83 \pm 6.77$ & $26.50 \pm 5.20$ & 0.002 \\
\hline Phonemic generative naming & $31.33 \pm 6.68$ & $26.17 \pm 7.78$ & $25.17 \pm 11.69$ & 0.379 \\
\hline \multicolumn{5}{|l|}{ Stroop test } \\
\hline Word & $111.83 \pm 0.41$ & $91.00 \pm 40.71$ & $103.67 \pm 14.95$ & 0.377 \\
\hline Color & $85.67 \pm 34.84$ & $82.33 \pm 36.01$ & $75.50 \pm 46.53$ & 0.353 \\
\hline
\end{tabular}

CDT = Clock Drawing Test; FU = follow-up; K-BNT = Korean version of the Boston Naming Test; RCFT = Rey Complex Figure Test; SVLT = Seoul Verbal Learning Test.

Values are expressed as least square mean \pm standard error.

dotomy with $45 \%-86 \%$ improvement. ${ }^{13,24,29}$ Conclusively, MRgFUS pallidotomy can achieve comparable effects of making lesions in deep brain tissue and consequent clinical results.

Unfortunately, we failed to increase the temperature sufficiently and make a visible lesion in 2 patients. Considering these unsatisfactory results related to temperature increase and permanent lesioning, several studies have focused on improving the delivery of sufficient ultrasonic energy through the skull. Chang et al. suggested that skull volume $\left(<320 \mathrm{~cm}^{3}\right)$ and skull density ratio $(>0.45)$ were significant factors for improving ultrasonic penetration of the skull, resulting in adequate lesioning and improved outcomes. ${ }^{8}$ In the present study, most patients satisfied skull density ratio and volume criteria, which were stricter than those utilized in previous ET studies. Nevertheless, we could not achieve adequate lesioning in some cases, and these findings suggest that additional factors may influence the distribution of ultrasonic energy by the skull. The heterogeneity of the individual skull composition, or of the ray angle to the skull, may present obstacles to increasing energy toward the target, even though these fac- tors have not been statistically verified in a previous study. ${ }^{8}$ In particular, the GPi target is generally located more laterally than the thalamus or internal capsule, which requires a larger acoustic beam angle, and consequently reduced energy transmission during sonication. Due to these potential issues, current mechanical systems have limitations in terms of generalization. Further studies on the technical aspects and universal reference values for skull data are required to overcome the limitations.

In terms of the safety of MRgFUS ablation, previous clinical trials have already proven the theoretical safety of thalamotomy for ET, ${ }^{7,13,23}$ Among the reported complications related to MRgFUS ablation, Elias et al. reported a case of dysesthesia in a patient's index finger. ${ }^{13}$ In our study, the safety of MRgFUS ablation was confirmed in most cases. With a stepwise sonication method, real-time monitoring, and a thorough neurological evaluation during the procedure, we safely and effectively created lesions and achieved symptomatic improvements without any significant side effects. Only 1 patient experienced acute neurological change during sonication, and we immediately stopped the procedure and addressed the situation when we 


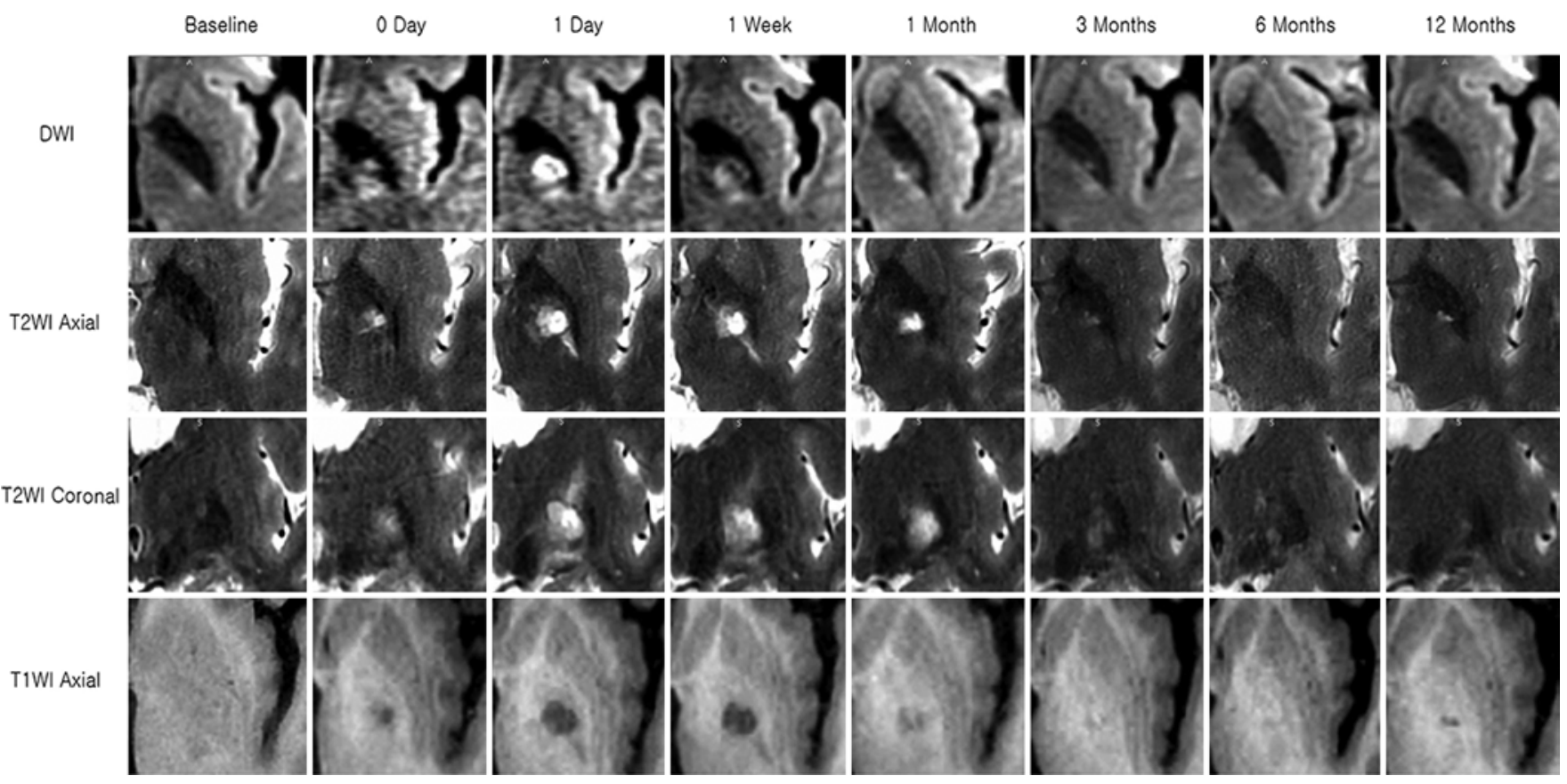

FIG. 2. Case 1. Changes in ablative lesions on conventional 3-T MR images after unilateral MRgFUS pallidotomy. The lesion size of the left pallidotomy was maintained until 1 month after MRgFUS. DWI = diffusion-weighted imaging; T1WI = T1-weighted imaging; $\mathrm{T} 2 \mathrm{Wl}=\mathrm{T} 2$-weighted imaging.

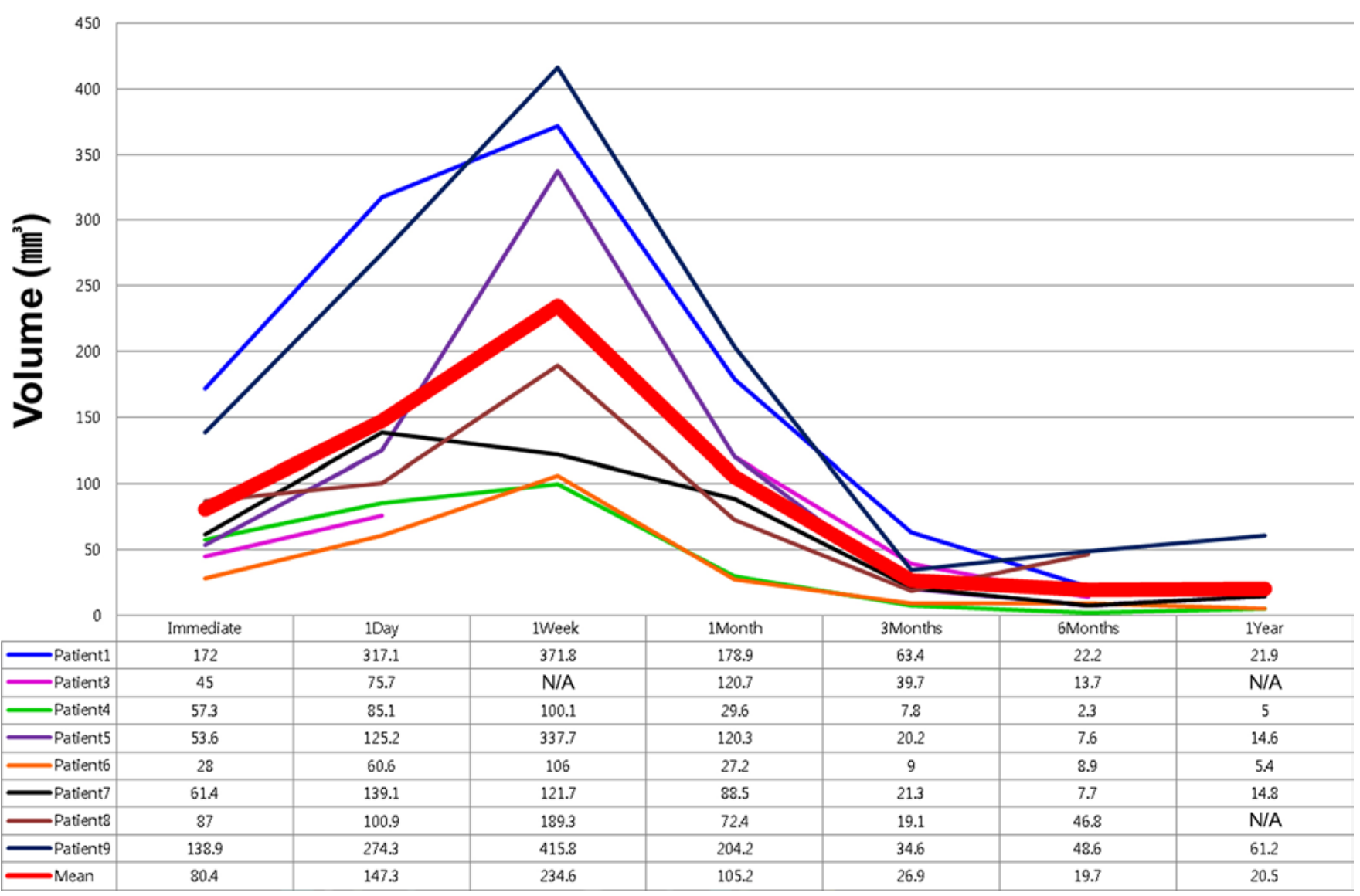

FIG. 3. Volume change of lesions after MRgFUS pallidotomy measured using GammaPlan software (10.0.0, Elekta AB). The extent of volume change from baseline to follow-up imaging was significant at 1 week after MRgFUS ablation. $N / A=$ not assessable as follow-up images were not acquired. Figure is available in color online only. 

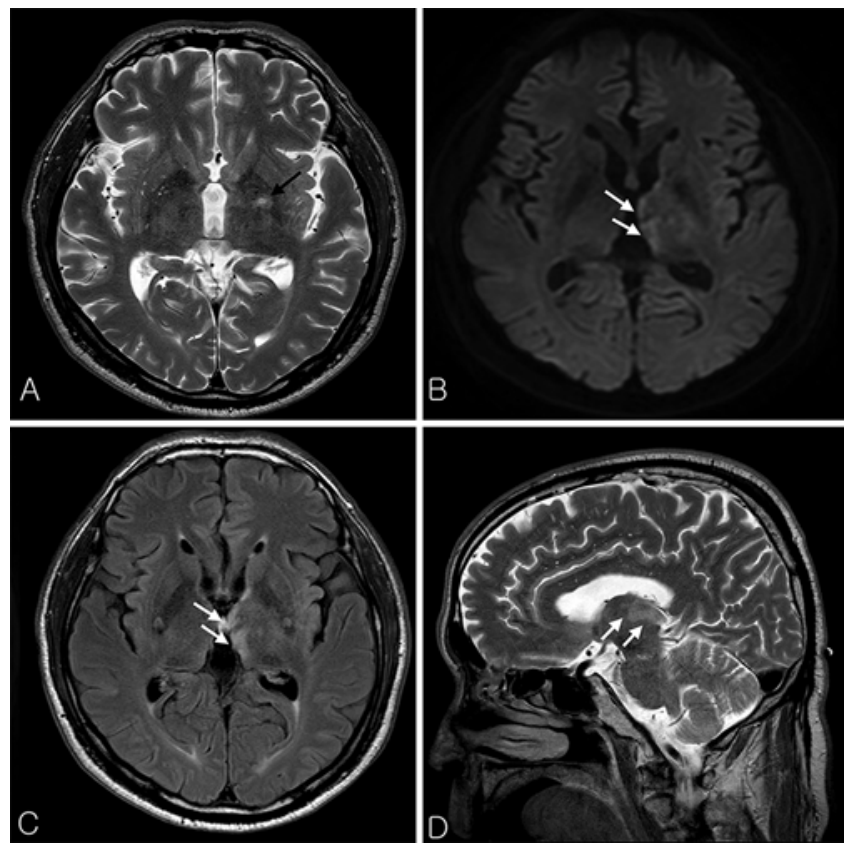

FIG. 4. Case 8. A case of heating injury of remote structures (thalamus and internal capsule) during sonication. Although a visible lesion was identified on T2-weighted images following left pallidotomy (black arrow, A), an abnormal high signal intensity was detected in the left thalamus (white arrows) on axial diffusion-weighted (B) and FLAIR images (C), and on sagittal T2-weighted images (D), which were obtained 1 day after MRgFUS.

observed the patient's change. Fortunately, his symptoms resolved completely within a few days. Several hypotheses have been proposed to explain this unexpected phenomenon. Small calcifications in the basal ganglia, which were detected on preoperative imaging, may have scattered ultrasonic energy, such that remote structures were affected. Another possible mechanism is that acoustically generated, inertial cavitation may have produced nonthermal biological effects on brain tissue. ${ }^{3}$ Moreover, many questions related to in vivo temperature increase remain unanswered..$^{32}$ Additional basic investigations are required to understand the characteristics of ultrasound and to confirm its safety. In terms of neuropsychological aspects, there were minor declines in some domains involving visual memory function and frontal executive function. Cognitive impairments are frequently encountered in advanced stages of PD; it cannot be determined whether cognitive changes were associated with lesioning by MRgFUS or the nature of PD itself. ${ }^{1} \mathrm{Re}-$ gardless of the change in cognition, patients' quality of life scores improved after the procedure.

\section{Limitations}

We acknowledge that the small number of patients, lack of randomization, and absence of a control group are limitations to the present study. The lack of statistical significance was likely because the study was underpowered; it would be meaningful to confirm feasibility of the new target using MRgFUS. A larger sample size and long-term follow-up are necessary to create a platform for expanding the scope of application.

\section{Conclusions}

The present study is the first trial involving patients with dyskinesia-dominant PD who underwent unilateral MRgFUS pallidotomy. Our findings demonstrated the feasibility of creating lesions at pallidal targets by means of MRgFUS and hold promise that MRgFUS pallidotomy can be used to control cardinal motor symptoms in patients with PD. However, further investigation and continuous follow-up are necessary to clarify the indications for MRgFUS pallidotomy, along with its safety and long-term effectiveness. Technical advances in MRgFUS are also required to make it possible to expand the indications for this lesioning approach, and finally to form the basis for a new direction in neurosurgery.

\section{Acknowledgments}

Itay Rachmilevitch and Eyal Zadicario, employees of InSightec (Haifa, Israel), provided excellent technical assistance and description of methods. The authors also thank Ms. Eun Jeong Kweon, $\mathrm{RN}$, for providing clinical assistance.

\section{References}

1. Aarsland D, Creese B, Politis M, Chaudhuri KR, ffytche DH, Weintraub D, et al: Cognitive decline in Parkinson disease. Nat Rev Neurol 13:217-231, 2017

2. Alkhani A, Lozano AM: Pallidotomy for Parkinson disease: a review of contemporary literature. J Neurosurg 94:43-49, 2001

3. American Institute of Ultrasound in Medicine Bioeffects Committee: Bioeffects considerations for the safety of diagnostic ultrasound. J Ultrasound Med 7 (9 Suppl):S1-S38, 1988

4. Blomstedt P, Hariz MI: Are complications less common in deep brain stimulation than in ablative procedures for movement disorders? Stereotact Funct Neurosurg 84:72-81, 2006

5. Bond AE, Dallapiazza R, Huss D, Warren AL, Sperling $\mathrm{S}$, Gwinn R, et al: A randomized, sham-controlled trial of transcranial magnetic resonance-guided focused ultrasound thalamotomy trial for the treatment of tremor-dominant, idiopathic Parkinson disease. Neurosurgery 63 (Suppl 1):154, 2016

6. Bulluss KJ, Pereira EA, Joint C, Aziz TZ: Pallidotomy after chronic deep brain stimulation. Neurosurg Focus 35(5):E5, 2013

7. Chang WS, Jung HH, Kweon EJ, Zadicario E, Rachmilevitch I, Chang JW: Unilateral magnetic resonance guided focused ultrasound thalamotomy for essential tremor: practices and clinicoradiological outcomes. J Neurol Neurosurg Psychiatry 86:257-264, 2015

8. Chang WS, Jung HH, Zadicario E, Rachmilevitch I, Tlusty T, Vitek S, et al: Factors associated with successful magnetic resonance-guided focused ultrasound treatment: efficiency of acoustic energy delivery through the skull. J Neurosurg 124:411-416, 2016

9. Chung SJ, Hong SH, Kim SR, Lee MC, Jeon SR: Efficacy and safety of simultaneous bilateral pallidotomy in advanced Parkinson's disease. Eur Neurol 56:113-118, 2006

10. de Bie RM, de Haan RJ, Nijssen PC, Rutgers AW, Beute GN, Bosch DA, et al: Unilateral pallidotomy in Parkinson's disease: a randomised, single-blind, multicentre trial. Lancet 354:1665-1669, 1999

11. Dewhirst MW, Viglianti BL, Lora-Michiels M, Hanson M, Hoopes PJ: Basic principles of thermal dosimetry and ther- 
mal thresholds for tissue damage from hyperthermia. Int J Hyperthermia 19:267-294, 2003

12. Dobrakowski PP, Machowska-Majchrzak AK, Labuz-Roszak B, Majchrzak KG, Kluczewska E, Pierzchała KB: MR-guided focused ultrasound: a new generation treatment of Parkinson's disease, essential tremor and neuropathic pain. Interv Neuroradiol 20:275-282, 2014

13. Elias WJ, Lipsman N, Ondo WG, Ghanouni P, Kim YG, Lee $\mathrm{W}$, et al: A randomized trial of focused ultrasound thalamotomy for essential tremor. N Engl J Med 375:730-739, 2016

14. Fine J, Duff J, Chen R, Chir B, Hutchison W, Lozano AM, et al: Long-term follow-up of unilateral pallidotomy in advanced Parkinson's disease. N Engl J Med 342:1708-1714, 2000

15. Fox SH, Katzenschlager R, Lim SY, Ravina B, Seppi K, Coelho M, et al: The Movement Disorder Society evidencebased medicine review update: treatments for the motor symptoms of Parkinson's disease. Mov Disord 26 (Suppl 3):S2-S41, 2011

16. Hua Z, Guodong G, Qinchuan L, Yaqun Z, Qinfen W, Xuelian W: Analysis of complications of radiofrequency pallidotomy. Neurosurgery 52:89-101, 2003

17. Jeanmonod D, Werner B, Morel A, Michels L, Zadicario E, Schiff G, et al: Transcranial magnetic resonance imagingguided focused ultrasound: noninvasive central lateral thalamotomy for chronic neuropathic pain. Neurosurg Focus 32(1):E1, 2012

18. Jenne JW: Non-invasive transcranial brain ablation with high-intensity focused ultrasound. Front Neurol Neurosci 36:94-105, 2015

19. Jung HH, Kim SJ, Roh D, Chang JG, Chang WS, Kweon EJ, et al: Bilateral thermal capsulotomy with MR-guided focused ultrasound for patients with treatment-refractory obsessivecompulsive disorder: a proof-of-concept study. Mol Psychiatry 20:1205-1211, 2015

20. Krack P, Martinez-Fernandez R, Del Alamo M, Obeso JA: Current applications and limitations of surgical treatments for movement disorders. Mov Disord 32:36-52, 2017

21. Laitinen LV, Bergenheim AT, Hariz MI: Leksell's posteroventral pallidotomy in the treatment of Parkinson's disease. J Neurosurg 76:53-61, 1992

22. Lang AE, Lozano AM, Montgomery E, Duff J, Tasker R, Hutchinson W: Posteroventral medial pallidotomy in advanced Parkinson's disease. N Engl J Med 337:1036-1042, 1997

23. Lipsman N, Schwartz ML, Huang Y, Lee L, Sankar T, Chapman M, et al: MR-guided focused ultrasound thalamotomy for essential tremor: a proof-of-concept study. Lancet Neurol 12:462-468, 2013

24. Lozano AM, Lang AE, Galvez-Jimenez N, Miyasaki J, Duff J, Hutchinson WD, et al: Effect of GPi pallidotomy on motor function in Parkinson's disease. Lancet 346:1383-1387, 1995

25. Magara A, Bühler R, Moser D, Kowalski M, Pourtehrani P, Jeanmonod D: First experience with MR-guided focused ultrasound in the treatment of Parkinson's disease. J Ther Ultrasound 2:11, 2014

26. Martin E, Jeanmonod D, Morel A, Zadicario E, Werner B: High-intensity focused ultrasound for noninvasive functional neurosurgery. Ann Neurol 66:858-861, 2009
27. Merello M, Nouzeilles MI, Kuzis G, Cammarota A, Sabe $\mathrm{L}$, Betti $\mathrm{O}$, et al: Unilateral radiofrequency lesion versus electrostimulation of posteroventral pallidum: a prospective randomized comparison. Mov Disord 14:50-56, 1999

28. Moser D, Zadicario E, Schiff G, Jeanmonod D: MR-guided focused ultrasound technique in functional neurosurgery: targeting accuracy. J Ther Ultrasound 1:3, 2013

29. Munhoz RP, Cerasa A, Okun MS: Surgical treatment of dyskinesia in Parkinson's disease. Front Neurol 5:65, 2014

30. Na YC, Chang WS, Jung HH, Kweon EJ, Chang JW: Unilateral magnetic resonance-guided focused ultrasound pallidotomy for Parkinson disease. Neurology 85:549-551, 2015

31. Nakamura K, Christine CW, Starr PA, Marks WJ Jr: Effects of unilateral subthalamic and pallidal deep brain stimulation on fine motor functions in Parkinson's disease. Mov Disord 22:619-626, 2007

32. O'Brien WD Jr: Ultrasound-biophysics mechanisms. Prog Biophys Mol Biol 93:212-255, 2007

33. Sapareto SA, Dewey WC: Thermal dose determination in cancer therapy. Int J Radiat Oncol Biol Phys 10:787-800, 1984

34. Spindola B, Leite MA, Orsini M, Fonoff E, Landeiro JA, Pessoa BL: Ablative surgery for Parkinson's disease: Is there still a role for pallidotomy in the deep brain stimulation era? Clin Neurol Neurosurg 158:33-39, 2017

35. Vitek JL, Bakay RA, Freeman A, Evatt M, Green J, McDonald W, et al: Randomized trial of pallidotomy versus medical therapy for Parkinson's disease. Ann Neurol 53:558-569, 2003

36. Vykhodtseva N, Sorrentino V, Jolesz FA, Bronson RT, Hynynen K: MRI detection of the thermal effects of focused ultrasound on the brain. Ultrasound Med Biol 26:871-880, 2000

37. Wu Z, Kumon RE, Laughner JI, Efimov IR, Deng CX: Electrophysiological changes correlated with temperature increases induced by high-intensity focused ultrasound ablation. Ultrasound Med Biol 41:432-448, 2015

\section{Disclosures}

Dr. Chang: grants from the Michael J. Fox Foundation, Focused Ultrasound Surgery Foundation, and other funding from InSightec.

\section{Author Contributions}

Conception and design: Chang. Acquisition of data: Jung, Park, Kim, Lee. Analysis and interpretation of data: Jung, Park, Kim. Drafting the article: Chang, Jung. Critically revising the article: Chang. Reviewed submitted version of manuscript: Lee, Sohn. Administrative/technical/material support: Chang, Lee, Sohn.

\section{Correspondence}

Jin Woo Chang: Yonsei University College of Medicine, Seoul, Korea.jchang@yuhs.ac. 